\title{
Localization of sound in the vertical plane with and without high-frequency spectral cues
}

\author{
ROBERT A. BUTLER and RICHARD A. HUMANSKI \\ University of Chicago, Chicago, Illinois
}

\begin{abstract}
Binaural localization of 3.0-kHz high- and lowpass noise presented in the median vertical plane (MVP) and lateral vertical plane (LVP) was investigated. We anticipated superior performance when localizing the highpass noise by virtue of the availability of pinna cues. The viability of this supposition was strengthened by monaural localization tests in which performance proficiency for the highpass noise exceeded that for the lowpass noise $(p<.01)$. The main result showed that binaural localization of proficiency for highpass noise surpassed that for lowpass noise for all listening conditions $(p<.01)$. However, the importance of binaural temporal and level differences in vertical-plane localization was demonstrated by the highly respectable performances when the lowpass noise was presented in the LVP. Data from binaural localization in the MVP and monaural localization in the LVP suggested that the influence of pinna cues diminishes for source elevations above $45^{\circ}$.
\end{abstract}

Behavioral research on localization of sound in space became moribund for many years following the publication of Stevens and Newman's (1936) study, for their data brought about a coalescence of two leading theories of sound localization; namely, binaural phase differences and binaural intensity differences. Both theories were called upon to adequately explain their results. Specifically, binaural phase differences were implicated in localizing lowfrequency sounds; binaural intensity differences were implicated in localizing high-frequency sounds. The large theoretical issue appeared to be settled. True, Mills (1958) contributed fundamental data on minimum audible angle, but for the most part, no new information leading to a more thorough understanding of sound localization was forthcoming. Psychologists turned to the lateralization paradigm for greater precision in substantiating the "duplex theory" of sound localization; neuroanatomists and neurophysiologists sought for the biological mechanisms whose functions were predicted by the theory.

Some 30 years after the Stevens and Newman (1936) paper, a revival of behavioral research on sound localization took place; the pinna's role in localizing sound, which by and large had lain dormant since Bloch's (1893) study, was resuscitated. Batteau's (1967) and Blauert's $(1969 / 70)$ ideas were primarily responsible for the renewed interest in the function of the pinna. Under the assumption that no binaural difference cues were available to guide location judgments, researchers began to study sound localization in the median vertical plane (MVP). And indeed, the importance of the pinna as a source of

This research was supported in part by NIH Grant R01 NS25889-02. Richard A. Humanski is with the Departments of Ophthalmology and Psychology. Address correspondence to Robert A. Butler, Departments of Surgery and Psychology, University of Chicago, Chicago, Il 60637. spectral cues was verified experimentally. Through its interaction with the higher audio frequencies of the incident sound wave, the pinna appears to function as a directionally dependent filter (Shaw, 1982). Distort them, occlude their cavities, or attenuate the high frequencies from the sound source, and localization performance in the MVP deteriorates precipitously (Gardner \& Gardner, 1973; Roffler \& Butler, 1968). Other data demonstrated that the pinna's role was not restricted to localization in the MVP. Manipulating the spectral properties of a sound placed in the lateral vertical plane (LVP) created, in monaural listeners, an illusion of changes in the sound's elevation (Bloom, 1977).

From this body of research emerged the idea that spectral cues furnished primarily by the pinna (a monaural contribution) were largely responsible for localizing sound in the vertical plane and that binaural difference cues were relegated to localizing sound in the horizontal plane. We have espoused this view, and not without reason. Humanski and Butler (1988) demonstrated that localization of sound in the LVP was as accurate when listening with only the ear nearest the sound source as it was when listening with both ears. In light of more recent data, it is imperative to point out that in the Humanski and Butler study, the loudspeakers covered a VP segment of only $\pm 30^{\circ}$ re interaural axis. Nonetheless, their results implied that monaural spectral cues were as adequate for guiding location judgments in the VP as were binaural difference cues. A recent study by Butler, Humanski, and Musicant (1990), however, points toward a major role of binaural difference cues in VP localization. Broadband noise was delivered by loudspeakers that extended from $0^{\circ}$ to $180^{\circ}$ azimuth and from $-45^{\circ}$ to $60^{\circ}$ elevation. Binaural localization of the VP-placed sounds was significantly more accurate than monaural localization. These investigators observed that monaural accuracy decreased progressively 
as the sound's elevation increased from $0^{\circ}$ to $60^{\circ}$. Since binaural localization remained reasonably proficient at the higher elevations, binaural differences must have been operative. Oldfield and Parker (1986) had reported a binaural superiority over monaural localization, but their effect was confined to the frontal region of the hemifield. It should be mentioned that in their study, loudspeakers were positioned no higher than $40^{\circ}$ above ear level. This truncated representation of the VP may have accounted for a failure to find a significant difference between binaural and monaural proficiency throughout the entire hemifield. Morimoto and Aokata (1984), while not comparing binaural with monaural performance, did report that binaural listeners accurately plotted the elevation component of sounds in the LVP-sounds that presumably were impoverished of monaural spectral cues since the upper cutoff frequency was $4.9 \mathrm{kHz}$ with a $60 \mathrm{~dB} /$ octave rejection slope. But whether that stimulus failed to provide even the rudiments of a monaural spectral cue was not tested directly.

In the present experiment, localization proficiency for 3.0-kHz high- and lowpass noise originating in the MVP and LVP was examined. We assumed that the highpass noise interacting with the contours of the pinnae would provide a greater degree of spectral information than would the lowpass noise; hence, localization of the former stimuli would be more accurate. We knew that binaural MVP localization required the presence of the higher audio frequencies. We assumed that listeners could accurately localize highpass noise in the LVP since both monaural spectral cues and binaural temporal and level difference cues would be available. What we did not know was how well listeners could binaurally localize lowpass noise in the LVP. With the absence of pinna cues, would binaural temporal and level difference cues serve to maintain accurate performance? We reasoned that the absence of useful spectral cues could be ascertained by demonstrating that monaural localization performance on the lowpass noise did not differ from chance expectancy, but monaural localization of highpass noise did. In light of the earlier studies from this laboratory (Butler et al., 1990; Humanski \& Butler, 1988), we anticipated that the monaural localization of highpass noise, while proficient at the lower elevations of the sound source, would be less so with increases in elevation of the sound source.

\section{METHOD}

Six listeners participated. Their auditory thresholds were within $10 \mathrm{~dB}$ of audiometric zero (ANSI, 1970) for frequencies ranging from $0.25 \mathrm{kHz}$ through $8.0 \mathrm{kHz}$. Their task was to report, via an intercom, which of seven loudspeakers was delivering the sounds. The loudspeakers (Realistic, Midrange Tweeters) were chosen from a pool of loudspeakers on the basis of comparable frequency response curves that were further smoothed by means of an equalizer (Rane, Model PE 15). They were positioned in the VP extending from $0^{\circ}$ re interaural axis to $90^{\circ}$ (overhead) and spaced $15^{\circ}$ apart, center to center. Supported by a pipe bent to specifications, each loudspeaker was fixed approximately $1.2 \mathrm{~m}$ from the center of the listener's head. That loudspeaker fixed at $0^{\circ}$ elevation was assigned the number 1 ; that stationed at $90^{\circ}$ was assigned the number 7 .
Table 1

The Various Conditions Under Which Localization Performances Were Investigated

\begin{tabular}{cccc}
\hline $\begin{array}{c}\text { Listening } \\
\text { Condition }\end{array}$ & $\begin{array}{c}\text { Listening } \\
\text { Mode }\end{array}$ & $\begin{array}{c}\text { Vertical-Plane } \\
\text { Location }\end{array}$ & \multicolumn{1}{c}{ Stimulus } \\
\hline 1 & Binaural & Lateral & $3.0-\mathrm{kHz}$ lowpass \\
2 & Binaural & Lateral & $3.0-\mathrm{kHz}$ highpass \\
3 & Binaural & Median & $3.0-\mathrm{kHz}$ lowpass \\
4 & Binaural & Median & $3.0-\mathrm{kHz}$ highpass \\
5 & Monaural & Lateral & $3.0-\mathrm{kHz}$ lowpass \\
6 & Monaural & Lateral & $3.0-\mathrm{kHz}$ highpass \\
\hline
\end{tabular}

The intervening loudspeakers were numbered in ascending order, 2 through 6. The auditory stimuli consisted of noise bursts, 100 msec in duration with a rise-decay time of $10 \mathrm{msec}$ and an interstimulus interval of $300 \mathrm{msec}$. Prior to presentation, the noise bursts were delivered to three filters (Krohn Hite, Model 3202 R) connected in series and set at either 3.0-kHz highpass or 3.0-kHz lowpass. Cascading the three filters resulted in a $72-\mathrm{dB} /$ octave rejection slope.

Seventy localization trials were given during a test session. Each loudspeaker delivered the noise bursts 10 times in an irregular order. Six listening conditions were established and are listed in Table 1. Presentation order of the six conditions followed a Latin square arrangement with rows representing listeners, columns representing test sessions, and numbers within the matrix representing listening conditions. The subjects were seated in a rotatable chair equipped with a headrest. They were cautioned not to move their heads during the test session. The experimenter, located in an adjacent room, could visually monitor the listeners' movements. Rarely did the listeners have to be reminded of the no-movement edict. All testing was conducted in a sound-treated room, $4.8 \times 4.1 \times 2.4 \mathrm{~m}$. For Conditions 1 and 2 (see Table 1), the loudspeaker column was fixed in the listeners' left hemifield at $90^{\circ}$ off midline (LVP). For Conditions 3 and 4, the listeners faced the loudspeaker column (MVP). For Conditions 5 and 6, the loudspeakers were again stationed in the LVP of the listeners' left hemifield. When listening monaurally (Conditions 5 and 6), the far (right) ear was occluded with ear mold material (Audi-Sil) and then covered with an ear muff (Bilsom Viking 2318) to achieve even greater attenuation. One test session was devoted to each condition.

The sounds were presented at approximately $25 \mathrm{~dB}$ sensation level, an approximation that was met in the following manner: $\mathrm{Be}$ fore each test session, thresholds for the scheduled noise burst were obtained under the appropriate listening condition for loudspeakers 1,4 , and 7. Thresholds for the intervening loudspeakers were estimated through interpolation. Intermingled in the trial sequence were level changes of $\pm 3 \mathrm{~dB}$ (frequently resulting in a 6-dB change from trial to trial) to discourage listeners from relying on relative loudness as a cue for location, false though it may have been. No verbal feedback was given to the subjects.

Localization proficiency was evaluated in terms of error score (ES). When a listener correctly identified the loudspeaker delivering the noise bursts, an ES of 0 was recorded. If the listener reported a loudspeaker 1,2 , or 3 loudspeakers removed from the correct one as the sound source, an ES of 1, 2, or 3 was recorded. Each test session yielded a single mean error score measurement, averaged across 70 trials. Consequently, a $t$ test was employed to evaluate the significance of mean ES differences between a priori pairwise comparisons of listening conditions. As multiple comparisons were performed, the test statistic was corrected by the method of Bonferroni.

\section{RESULTS}

Proficient performances were observed when the listeners binaurally localized the $3.0-\mathrm{kHz}$ lowpass noise 
bursts originating in the LVP (Condition 1), but poor performances were observed when the listeners binaurally localized the same signal originating in the MVP (Condition 3 ). The difference in performance accuracy between Conditions 1 and 3 was significant at the 0.01 level (see Figure 1, described below). These two listening conditions, differentiated by source location, correspondingly differed in the availability of binaural time and level information; in Condition 1, binaural information was available. In Condition 3 , the only possibly binaural cue would have been that furnished by a disparity in the frequency response of the pinnae-a cue, however, that emerges only at the higher stimulus frequencies (see Searle, Braida, Cuddy, \& Davis, 1975). ${ }^{1}$ When contrasting performance across the same source locations for the highpass stimuli (i.e., compare Conditions 2 and 4), qualitatively similar differences were observed $(p<.01)$ but the magnitude of the effect was markedly reduced. The decreased magnitude of the difference in performance across source locations presumably resulted from enhanced baseline levels of performance owing to the availability of monaural spectral cues.

The contributions yielded by binaural cues, previously demonstrated by contrasting source locations, are also shown by contrasting binaural listening conditions differentiated only by the frequency content of the stimuli. Such comparisons show that binaural localization of sound in the VP universally benefited from stimuli consisting of highpass noise relative to lowpass noise. This finding is demonstrated in Figure 1, which summarizes localization performance for three pairs of conditions differentiated only by whether the stimulus consisted of $3.0-\mathrm{kHz}$ highpass or lowpass noise. The open bars represent mean ES when localizing the lowpass noise bursts; the striped bars represent mean ES when localizing the highpass noise bursts. Standard error of the mean is shown above each bar. As is evident in Figure 1, in each case where binaural performance on high- and lowpass noise bursts are compared directly, that is, binaural localization in the LVP (Conditions 1 and 2) and binaural localization in the MVP (Conditions 3 and 4), performance proficiency for the highpass noise surpassed that recorded for the lowpass noise at significance levels $\geq .01$.

An issue unresolved by the prior comparisons is the extent to which the experimental factor " frequency content of the stimulus' isolated binaural cues for localization in the LVP. Monaural listening measurements obtained for low- and highpass stimuli in the LVP showed that isolation of binaural temporal and overall level difference cues in the LVP by employing lowpass stimuli was complete. As with those comparisons that focused on frequency content of the stimulus under binaural conditions, monaural localization performance improved $(p<.01)$ when the stimulus consisted of high versus low frequencies (see Figure 1, Conditions 5 and 6). Clearly, as listening was monaural, any observed differences are attributable exclusively to spectral cues, thereby underscoring the contribution of these cues toward accurately localizing vertically positioned sounds. Moreover, as performance for the lowpass condition failed to differ from chance levels, "frequency content of the stimulus" not merely degraded spectral cues, it eliminated them.

Insight with respect to the specific spatial regions across which the previously observed differences in localization of high- and lowpass noise arose can be obtained by viewing the results from a different perspective. This is done

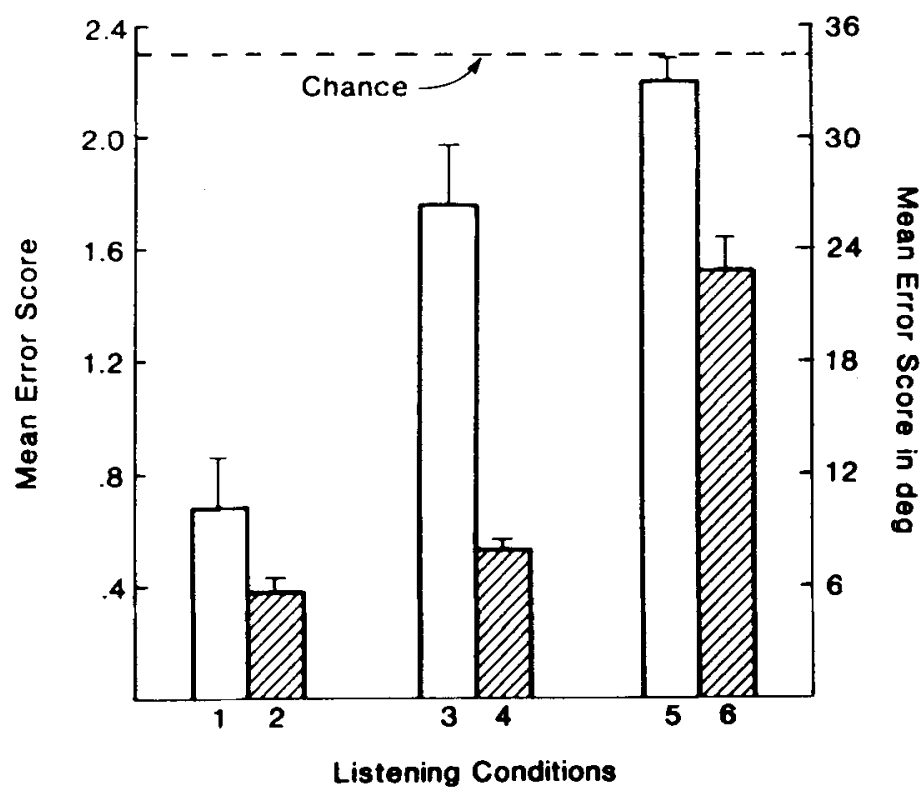

Figure 1. Mean error score calculated for the various listening conditions. See text for further detalls. 

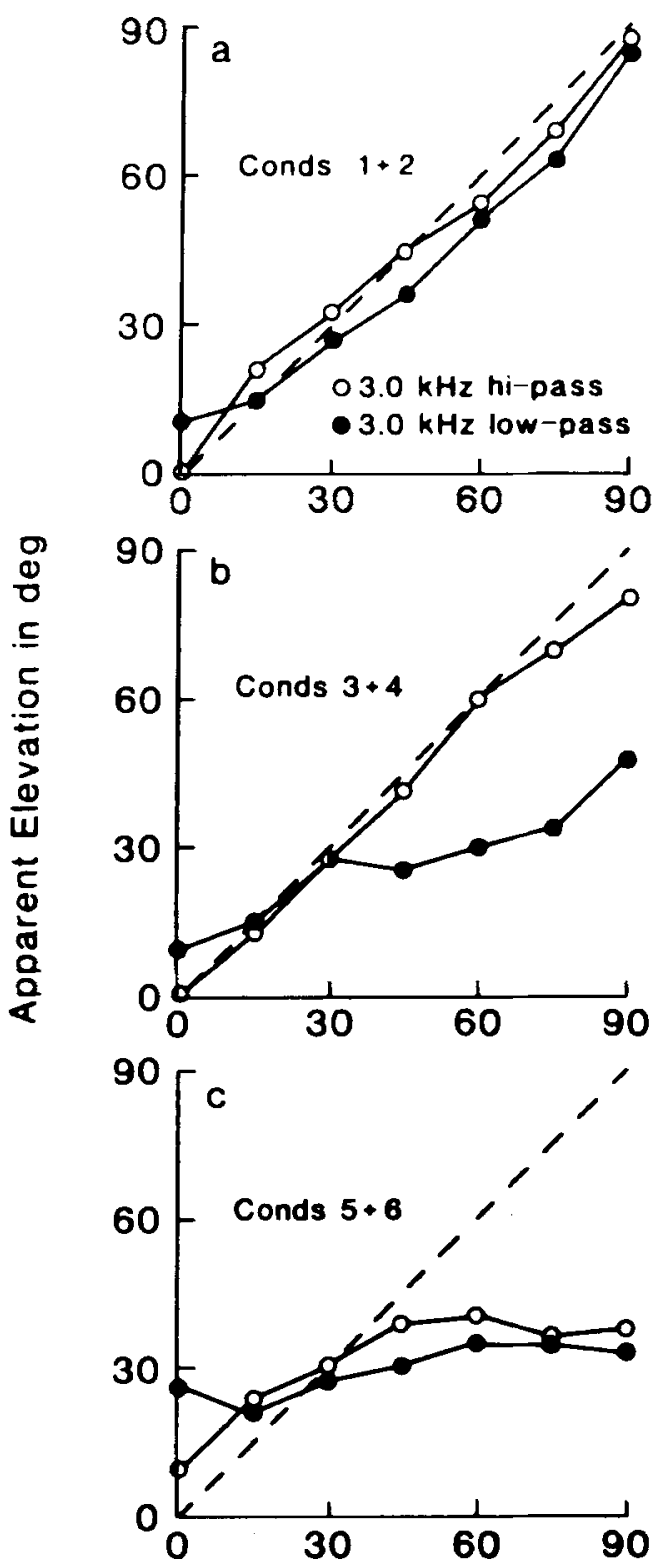

Actual Elevation in deg

Figure 2. Apparent elevation of the sound sources plotted against their actual elevations. See text for further details.

in Figure 2, where mean apparent location of the sound source is plotted against its actual location. The figure consists of three panels, each reporting results for pairs of conditions differentiated only by the frequency content of the stimulus. Open circles signify location judgments of the 3.0-kHz highpass noise bursts; closed circles signify location judgments of the $3.0-\mathrm{kHz}$ lowpass noise bursts. Consider first the performance data for Conditions 1 and 2 (Figure 2a). When the sounds originated in the LVP, mean perceived elevation of the noise bursts corresponded quite closely to their actual locations, although that for the lowpass noise deviated somewhat more from the sounds' actual locations at elevations of $45^{\circ}$ and above. Performance data for Conditions 3 and 4, conditions in which the sounds originated in the MVP, are shown in Figure $2 \mathrm{~b}$. The mean perceived elevation when the noise bursts contained the higher audio frequencies followed closely their actual elevation up to $60^{\circ}$ elevation. Mean perceived elevation of the lowpass noise departed abruptly from its actual elevation when the sounds were presented at elevations greater than $30^{\circ}$. Note that the context in which listeners localized the highpass noise originating directly overhead $\left(90^{\circ}\right.$ elevation) influenced location judgments. When the localization trials were presented in the LVP, listeners reported the sounds as originating at $90^{\circ}$ elevation; when the trials were presented in the MVP, listeners were more likely to report the sounds as coming from around $75^{\circ}$ elevation. In both instances, the origin of the stimuli was identical. Figure $2 c$ shows monaural performance data for the two differently filtered noise bursts. Neither were localized proficiently. Yet the apparent elevation of the $3.0-\mathrm{kHz}$ highpass noise bursts matched reasonably well with their actual elevation up to $45^{\circ}$. At higher elevations, localization performance deteriorated.

\section{DISCUSSION}

Implicit in the literature is the notion that the pinna is essential for localizing sounds in the VP. The pinna, because of its small size, can only affect differentially the acoustic energy of sounds with short wavelengths. It follows, then, that removal of the higher audio frequencies from the stimulus would result in an inability to localize VP-positioned sounds. This appears to be so when localizing monaurally. However, with the use of both ears, listeners can unquestionably localize sound devoid of pinna cues: note the high-level performances when localizing the lowpass noise originating in the LVP. Furthermore, the failure of monaural listeners to localize $3.0-\mathrm{kHz}$ lowpass sound better than chance confirms that monaural spectral cues were absent when the lowpass stimulus originated in the LVP. Consequently, the proficient binaural localization of the 3.0-kHz lowpass noise bursts emanating from the LVP was based solely on binaural time and level differences. Otherwise, it would be difficult to believe that an inadequate monaural spectral cue stemming from the near-ear pinna coupled with an even less adequate monaural spectral cue stemming from the far-ear pinna would contribute positively toward localization accuracy. While the pinna cues were not essential for proficient LVP performances, they did serve to improve accuracy as attested by significantly lower error scores for the highpass noise. In the MVP, on the other hand, pinna cues were critical for reasonably accurate performances, a finding consistent with previous research (Gardner \& Gardner, 1973; Roffler \& Butler, 1968).

In summary, the monaural data suggest that monaural spectral cues contribute toward attaining accuracy in localizing sound in the LVP up to approximately $45^{\circ}$ elevation. To localize with precision sounds distributed throughout the LVP requires the presence of binaural temporal 
and level information in addition to pinna cues. MVP localization, when supported by pinna disparity cues inherent in the higher frequencies; falters above $60^{\circ}$ elevation. As more and more behavioral data become available on localization in two-dimensional space, the duplex theory, which still serves as a guide for neurophysiology and neuroanatomical research in this area, is no longer adequate. Spectral cues in the form of binaural spectral differences, frequency by frequency or frequency band by frequency band, as well as a monaural spectral input must be integrated into a more comprehensive theory.

\section{REFERENCES}

ANSI (1970). Specifications for audiometers (ANSI S3.6-1969). New York: American National Standards Institute.

BAtTEAU, D. W. (1967). The role of the pinna in human localization. Proceedings of the Royal Society of London, 168, Series B, 158-190. BlaUERT, J. (1969/70). Sound localization in the median plane. Acustica, 22, 205-213.

ВLOCH, E. (1893). Das binaurale Horen. Zeitschrift fur Orhenheilkunde, $24,25-85$.

BLoom, P. J. (1977). Creating source elevation illusions by spectral manipulation. Journal of the Audio Engineering Society, 25, 560-565.

Butler, R. A., Humanski, R. A., \& Musicant, A. D. (1990). Binaural and monaural localization of sound in two-dimensional space. Perception, 19, 241-256.

Gardner, M. B., a Gardner, R. S. (1973). Problem of localization in the median plane. Journal of the Acoustical Society of America, $53,400-408$.

Humanski, R. A., Butler, R. A. (1988). The contribution of the near and far ear toward localization of sound in the sagittal plane. Journal of the Acoustical Society of America, 83, 2300-2310.
Milss, A. W. (1958). On the minimum audible angle. Journal of the Acoustical Society of America, 30, 237-246.

Morimoto, M., AokATA, H. (1984). Location cues of sound sources in the upper hemisphere. Journal of the Acoustical Society of Japan, $5,165-173$.

Oldfield, S. R., \& Parker, S. P. A. (1986). Acuity of sound localisation: A topology of auditory space. III. Monaural hearing conditions. Perception, 15, 67-81.

Roffler, S. K., \& Butler, R. A. (1968). Factors that influence the localization of sound in the vertical plane. Joumal of the Acoustical Society of America, 43, 1255-1259.

Searle, C. L., Braida, L. D., Cuddy, D. R., \& Davis, M. F. (1975). Binaural pinna disparity: Another auditory localization cue. Joumal of the Acoustical Society of America, 57, 448-455.

SHAw, E. A. G. (1982). External ear response and sound localization. In R. W. Gatehouse (Ed.), Localization of sound: Theory and applications (pp. 30-41). Groton, CT: Amphora.

Stevens, S. S., Newman, E. B. (1936). The localization of actual sources of sound. American Journal of Psychology, 48, 297-306.

\section{NOTE}

1. One listener localized, binaurally, the 3.0-kHz lowpass noise bursts originating in the MVP (Condition 1) with a high degree of accuracy that suggests that pinna disparity cues were available to her. When, at the conclusion of the study, she was binaurally presented a $2.0-\mathrm{kHz}$ lowpass noise stimulus, her performance (distribution of location judgments) failed to differ from chance expectation (chi-square test: $p>.05$ ). She was unable to localize the lowpass noise monaurally (Condition 5) as her distribution of location judgments also failed to differ from chance expectation (chi-square test: $p>.05$ ). Conceivably, pinna disparity cues operate at lower stimulus frequencies than do monaural spectral cues.

(Manuscript received February 4, 1991; revision accepted for publication September 27, 1991.) 